\title{
A yeast TFIIB-related factor involved in RNA polymerase III transcription
}

\author{
Trenton Colbert ${ }^{1,2}$ and Steven Hahn ${ }^{1}$ \\ ${ }^{1}$ Fred Hutchinson Cancer Research Center, Seattle, Washington 98104-2092 USA; ${ }^{2}$ Department of Pathology, University of \\ Washington, Seattle, Washington 98104-2092 USA
}

\begin{abstract}
A suppressor gene was identified, which in high copy number rescues a temperature-sensitive mutation in yeast TATA-binding protein (TBP). Suppression was allele specific because the suppressor did not rescue the temperature-sensitive phenotype of another TBP mutant. This suppressor gene encodes a 596-amino-acid protein of which the amino-terminal half is homologous to the Pol II-specific factor TFIB. Disruption of this gene, termed BRF1, showed it to be essential for growth of yeast. Deletion of sequences at either the amino or carboxyl terminus of BRF1 gave both temperature- and cold-sensitive phenotypes. These temperature- and cold-sensitive strains were used to prepare extracts deficient in BRF1 activity and were tested for transcriptional activity by RNA polymerases I, II, and III in vitro. BRF1-deficient extracts are defective in Pol III transcription and can be reconstituted for Pol III transcription by the addition of recombinant BRF1. Western analysis shows that BRF1 is present in TFIIIB but not the TFIIIC fraction, suggesting that it is a component of TFIIIB. We propose that BRF1 plays a role in Pol III initiation analogous to the role played by TFIB for Pol II in its interaction with TBP and polymerase. The identification of a Pol III-specific TFIIB-like factor extends the previously noted similarity of transcriptional initiation by the three nuclear polymerases.
\end{abstract}

[Key Words: Yeast TFIIB; TATA-binding protein; RNA Pol III; TFIIIB]

Received July 17, 1992; accepted August 4, 1992.

Accurate initiation of transcription by the three nuclear RNA polymerases, Pol I, Pol II, and Pol III, requires numerous general transcription factors in addition to the polymerase enzymes. These general factors serve in part to precisely position the polymerase enzyme at the transcription start site. There are at least seven general Pol II factors, TFIIA-TFIIJ, (for review, see Zawel and Reinberg 1992). Pol I requires at least two general factors, UBF and SLl (Learned et al. 1985; Bell et al. 1988; Sollner-Webb and Mougey 1991; Reeder 1992). Finally, Pol III requires three factors, TFIILA, TFIIIB, and TFIIIC /Geiduschek and Tocchini-Valentini 1988; Gabrielson and Sentenac 1991; Geiduschek and Kassavetis 1992). TFIIIA is required for transcription only from the 5S rRNA gene, and is composed of a single polypeptide (Ginsberg et al. 1984; Woychik and Young 1992). TFIIIB has been separated further into TFIIIB' and B', which have been correlated with proteins of 70 and $90 \mathrm{kD}$ by DNA cross-linking, but TFIII may contain other necessary polypeptides (Bartholomew et al. 1991; Kassavetis et al. 1991). TFIIIC contains multiple polypeptides, five of which can be crosslinked to the internal control region of the SUP4 tRNA gene (Braun et al. 1989; Bartholomew et al. 1990; Swanson et al. 1991; Parson and Weil 1992).

The three polymerase systems are, however, not completely distinct. First, five subunits are shared between the Pol I, Pol II, and Pol III enzymes (Woychik et al. 1990; Carles et al. 1991; Young 1991; for review, see Sentenac 1985). Second, at least one factor, the TATA-binding pro- tein (TBP), is required for transcription by all three polymerases (Lobo et al. 1991; Margottin et al. 1991; Simmen et al. 1991; Comai et al. 1992; Cormack and Struhl 1992; Schultz et al. 1992; White et al. 1992). These results suggest the possibility that some of the previously denoted polymerase-specific general factors could also be shared between the three polymerase systems. Alternatively, related but polymerase-specific general factors could exist that perform a similar function for each of the three polymerases.

We demonstrated previously that mutations in yeast TBP could differentially affect expression of the three polymerases in vitro (Schultz et al. 1992). One temperature-sensitive allele of TBP abolished Pol I, Pol II, and Pol III transcription. Another temperature-sensitive allele abolished only Pol II and Pol III transcription. A third dominant TBP mutation affected only transcription by Pol II. In this paper we describe a suppressor of the TBP mutant defective in Pol II and Pol III transcription. This suppressor encodes a factor that is required for maximal levels of transcription by Pol III and is similar in protein sequence to the Pol II-specific general factor TFIIB.

\section{Results \\ Isolation of an allele-specific suppressor of a temperature-sensitive TBP mutation}

We have characterized two temperature-sensitive alleles 
of yeast TBP (Schultz et al. 1992). Strains containing either of these mutant alleles grow normally at $25^{\circ} \mathrm{C}$, but grow slowly at $37^{\circ} \mathrm{C}$ (Fig. 1). One of these mutations, Pro-65 changed to Ser (P65S), was defective in Pol II and Pol III transcription in vitro. The other mutation, Il43N, was defective in all three nuclear RNA polymerase activities. To identify gene products that normally interact with TBP, we screened for genes, which when present in high-copy number could restore growth of the temperature-sensitive strains at $37^{\circ} \mathrm{C}$. This strategy has been used previously to identify interacting gene products such as cdc2 and suc1 (Hayles et al. 1986).

The two temperature-sensitive strains were transformed with a yeast genomic DNA library contained on a high-copy number $2 \mu$ vector. For the strain containing the I143N change, revertants at $37^{\circ} \mathrm{C}$ were found at a frequency of $\sim 1 / 400$ transformants. Ten of these revertants were analyzed, and all were found to contain the SPT15 gene encoding yeast TBP. For the P65S change, revertants were found at a frequency of $\sim 1 / 700$ transformants. Of 13 revertants analyzed, 8 contained the SPT15 gene and 5 contained overlapping DNA fragments that did not contain SPT15. Upon retransformation of the two temperature-sensitive strains with the non-SPT15 plasmids, it was observed that these plasmids suppressed the temperature-sensitive defect of only the P65S mutation and not the I143N mutation (Fig. 1, cf. strains A and $\mathrm{C}$ at $37^{\circ} \mathrm{C}$. When the P65S mutant strain contained the high-copy suppressor plasmid it grew at nearly the same rate as the wild-type strain at $37^{\circ} \mathrm{C}$ (data not shown).

To map the location of the suppressor gene, fragments of the plasmid containing the smallest genomic DNA

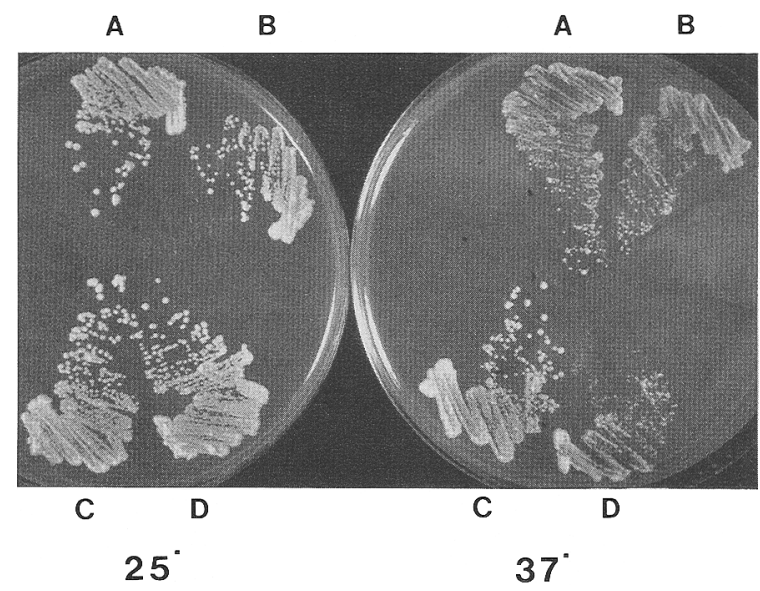

Figure 1. Allele-specific suppression of a temperature-sensitive TBP mutation. Yeast containing either of the two temperature-sensitive TBP mutations were transformed with the $2 \mu$ plasmid pSH256 containing the suppressor gene (BRF1) or transformed with the $2 \mu$ vector YEP24, which lacks a yeast genomic DNA insert. Cells were grown on minimal glucose plates lacking leucine and uracil at the indicated temperatures. (A) I143N mutant transformed with pSH256. (B) I143N mutant transformed with YEP24. (C) P65S mutant transformed with pSH256. (D) P65S mutant transformed with YEP24.

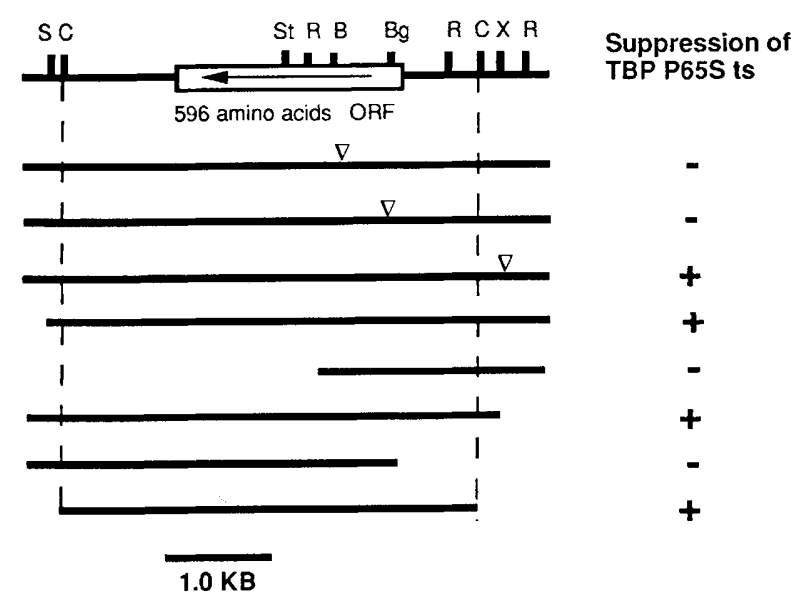

Figure 2. Localization of $B R F 1$-coding sequences. The indicated DNA fragments were subcloned to a high-copy $2 \mu$ plasmid and tested for suppression of the P65S TBP mutation at $37^{\circ} \mathrm{C}$. Frameshift mutations in $B R F 1$ were constructed by cutting and filling in overhanging sticky ends of $B a m H I, B g I I$, and $X$ hoI restriction sites as indicated. $(\mathrm{S}) \mathrm{SacI} ;(\mathrm{C}) \mathrm{ClaI} ;\{\mathrm{St}\rangle \mathrm{StuI},(\mathrm{R})$ EcoRI; (B) BglII; (X) XhoI.

insert were subcloned and tested for suppression of the P65S mutation. In addition, several frameshift mutations were constructed (Fig. 2). The suppressor activity was localized to a 4-kb ClaI DNA fragment. Most of this DNA fragment was sequenced and found to contain one large open reading frame (ORF) encoding a protein of 596 amino acids with a predicted molecular mass of $67 \mathrm{kD}$ (Fig. 3). Frameshift mutations within this ORF abolished the suppressor activity (Fig. 2).

The temperature-sensitive suppressor is a TFIIB-related factor

The amino acid sequence of the suppressor was compared with the available protein and translated nucleic acid sequence data bases. This analysis revealed that the amino-terminal half of the suppressor was homologous to the Pol II general transcription factor (TFIIIB. Below, we term the suppressor gene BRF1 for TFIIB-related factor. Alignment of amino acids $1-320$ of BRF1 with human TFIIB showed that the two sequences were $\sim 23 \%$ identical. One striking difference between BRF1 and the TFIIB factors is that BRF1 has at least 300 amino acids of carboxy-terminal sequence that is unrelated to TFIIB. This carboxy-terminal nonconserved region of BRF1 is highly charged but has no obvious similarity to other proteins in available data bases.

The genes encoding TFIIB have been cloned from human, Drosophila, Xenopus, and Saccharomyces cerevisiae (Ha et al. 1991; Hisatake et al. 1991; Malik et al. 1991; Pinto et al. 1992; Yamashita et al. 1992). The vertebrate and Drosophila proteins are all at least $80 \%$ identical, whereas the yeast protein (SUA7) is the most distantly related, with $35 \%$ identity to human TFIIB. The protein size varies from 316 to 345 amino acids in length. 


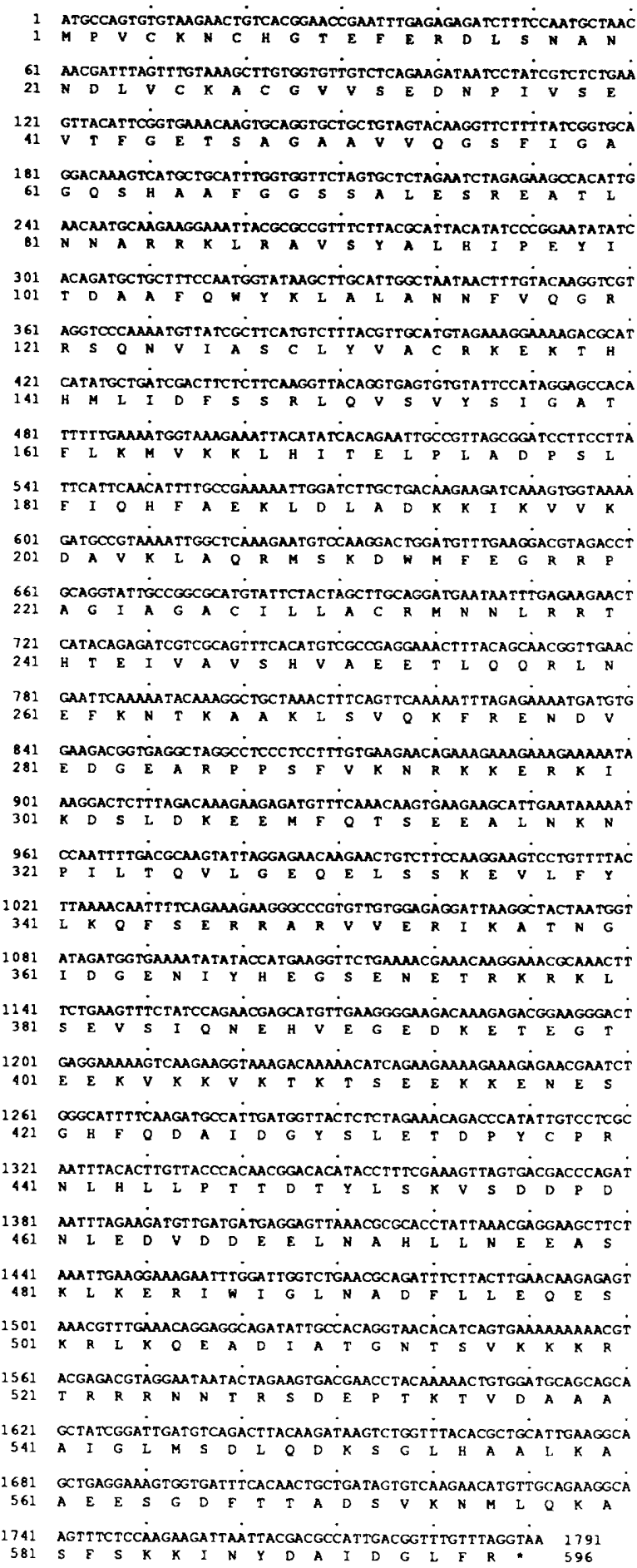

Figure 3. DNA sequence of $B R F 1$. The coding sequence and translated amino acid sequence of $B R F 1$ are shown. These sequences as well as $5^{\prime}$ - and $3^{\prime}$-noncoding DNA sequences are available in GenBank under accession no. M91073 and locus YSCTFIIBRF.

In Figure 4, the sequence of BRF1 is aligned with the cloned TFIIB sequences, and regions conserved between all five proteins are indicated. Residues that are identical or conservatively substituted in four of five of the pro- teins are indicated in boldface type in Figure 4B. The TFIIB-related family of genes contains three regions of conserved residues (denoted 1, 2, and 3 in Fig. 4). Region 1 is a 19-amino-acid sequence at the amino termini of the five proteins, hypothesized to be involved in metal binding (Pinto et al. 1992). Regions 2 and 3 contain the $\sim 100$-amino-acid imperfect direct repeat that has been noted previously by Malik et al. (1991). A detailed alignment of regions 1, 2, and 3 in Figure $4 \mathrm{~B}$ shows that four TFIIB proteins contain a high percentage of residues that are identical or similar. Comparison of BRF1 with the TFIIB proteins reveals that fewer residues are identical or similar, but BRF1 is clearly related to all of the TFIIB proteins. Interestingly, the charged amino acids at the end of the first repeat in region 2 , as well as the sequences between the repeats, are not conserved between BRF1 and the TFIIB factors. Note also that region 2 contains conserved sequences amino-terminal to the first repeat, which are stippled in Figure 4A.

To estimate the relatedness of the five TFIIB proteins, pairwise alignments of individual family members were compared to pairwise alignments where one of the protein sequences was randomly shuffled. The relatedness of two sequences is expressed as the number of standard deviations (s.D.s) by which the Lipman and Pearson alignment score differs from the mean alignment score of 100 alignments with a randomly shuffled sequence. The comparison to a shuffled sequence eliminates the inclusion of spurious similarities in the alignment score. Alignment scores $>6$ s.D.s above the mean shuffled score suggest that two sequences are related (Pearson 1990). Pairwise alignment of the human, fly, frog, and yeast proteins all show scores $>30$ s.D.s from the mean score of the random shuffle scores (see Fig. 4A). BRF1 is clearly related to the vertebrate and Drosophila TFIIB with a score of 17 . Interestingly, BRF1 is less related to yeast TFIIB (SUA7) with a score of 8 .

\section{BRF1 is essential for growth of yeast}

As detailed in Materials and methods, one copy of the $B R F 1$ gene was disrupted by recombination in a diploid strain. This disruption resulted in deletion of the conserved amino-terminal half of the $B R F 1$-coding sequence and insertion of the HIS4 gene. Sporulation of the diploid strain resulted in no $\mathrm{HIS}^{+}$spores $(0 / 24)$, suggesting that $B R F 1$ is an essential gene. To prove that BRF1 is not merely required for spore germination, a plasmid loss assay was done. Haploid strains were constructed with $B R F 1$ disrupted at the chromosomal locus and wild-type $B R F 1$ on a $U R A 3$-marked plasmid. Plating on 5-fluoroorotic acid (FOA), which selects against $\mathrm{Ura}^{+}$cells, produced no $\mathrm{Ura}^{-}$segregants unless the strain also contained another copy of $B R F 1$ on a separate $L E U 2$-marked plasmid. This proves that $B R F 1$ is an essential gene.

Southern analysis of wild-type and disrupted strains revealed that the $B R F 1$ probe also hybridized weakly to a second distinct genomic DNA fragment even when blots were washed at high stringency. This hybridization was 


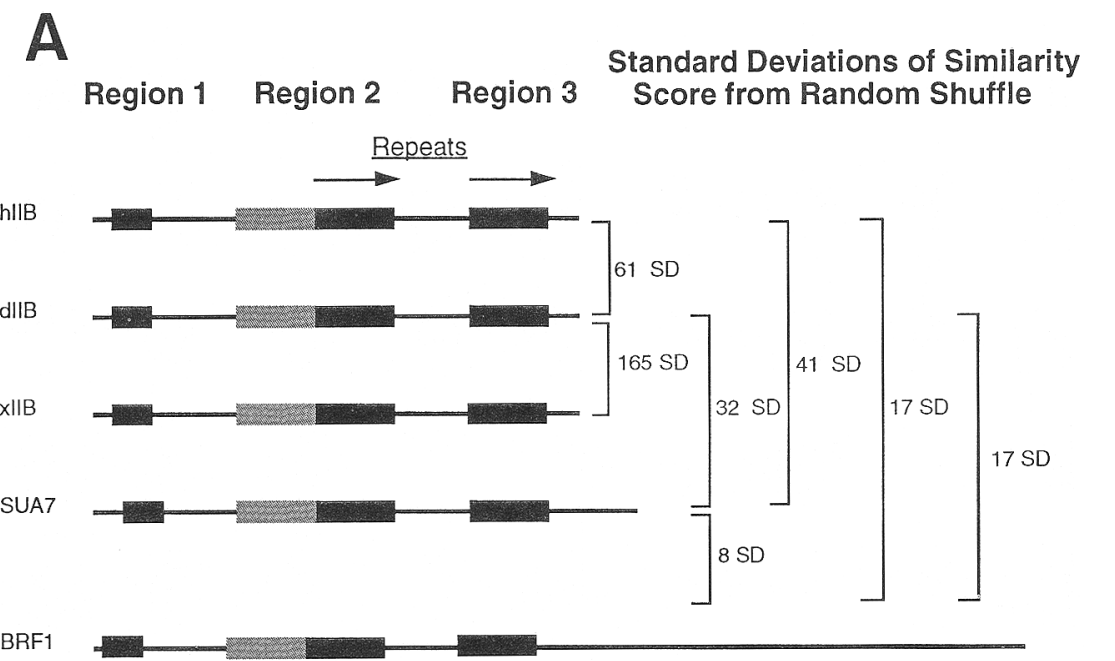

Figure 4. $B R F 1$ is similar to TFIIB. $|A|$ Graphic representation of the similarity between BRFl and four TFIIB protein sequences: Human TFIIB (hIIB), Drosophila IIB (dIIB), Xenopus IIB (xIIB), and yeast IIB (SUA7). (D) Blocks of similarity. Arrows indicate the location of the imperfect direct repeat. The positions of the blocks are intended to approximate their relative positions in the protein sequence. The overall similarity between pairs of proteins, indicated by standard deviations (S.D.), was estimated by the RSS program as described in Results and Materials and methods. $|B|$ Alignment of similar sequence blocks. Similar or identical residues in four of five proteins are in boldface type.

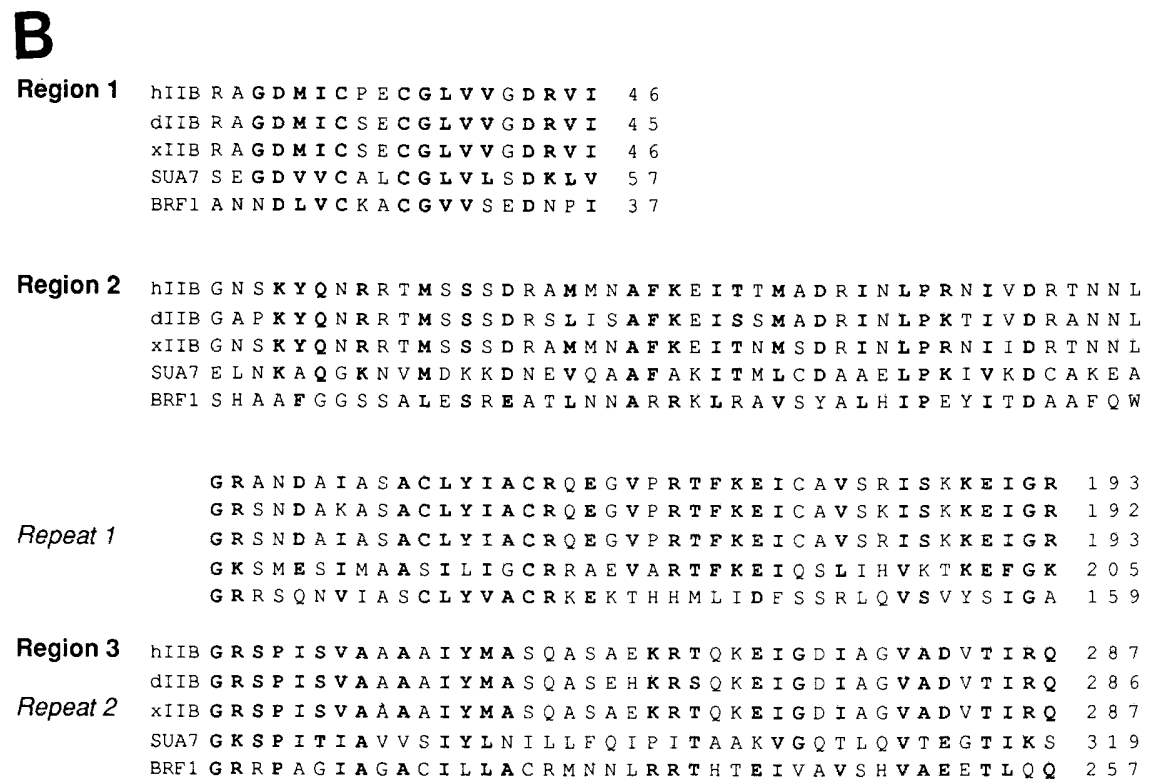

most strong using a probe encoding the conserved amino-terminal half of BRF1 (D. Auble, pers. comm.). Hybridization was not observed when using a $5^{\prime}$ noncoding sequence probe, and observed only weakly when using the nonconserved half of BRF1-coding sequences. This weak hybridization did not represent detection of the $S U A 7$ gene as determined using a $S U A 7$ probe. Whether this observation represents a pseudogene, a second copy of $B R F 1$, or a related gene involved in some other polymerase system remains to be determined.

\section{Deletion analysis of BRF1}

The conserved regions between the TFIIB-like family members suggest that these sequences have important structural or functional roles. To begin an analysis of the functional domains of BRF1, several deletions of the $B R F 1$ gene were constructed by PCR amplification. The deleted genes were substituted for the wild-type BRF1- coding sequence such that expression was driven by the wild-type BRF1 promoter. The viability of each deletion was tested by a plasmid shuffle assay (Fig. 5). Deletion of 10 or 25 amino acids from the amino terminus of BRF1 $(\Delta \mathrm{N} 10$ and $\Delta \mathrm{N} 25)$ gave viable cells that grew normally at $30^{\circ} \mathrm{C}$. The deletion of 25 amino acids from the amino terminus eliminates approximately half of the putative metal-binding domain described by Pinto et al. (1992). In contrast to the smaller deletions, the removal of 40 amino-terminal residues was lethal $(\Delta N 40)$. This deletion removed all of the conserved region 1 sequences. Likewise, deletion of 20 or 50 amino acids from the carboxyl terminus of BRF1 ( $\Delta \mathrm{C} 20$ and $\Delta \mathrm{C} 50)$ gave cells that grew normally at $30^{\circ} \mathrm{C}$. Deletion of 100 carboxy-terminal residues $(\Delta \mathrm{C} 100)$ was lethal. These results demonstrate that the nonconserved carboxy-terminal half of BRF1 has an essential function. Surprisingly, all four deletions that gave viable cells at $30^{\circ} \mathrm{C}$ gave both cold- and temperature-sensitive phenotypes (Fig. 5). Each deletion grew 
Figure 5. Viability and thermal sensitivity of the strains harboring fulllength or deleted variants of BRF1. $(A)$ Thermal sensitivity of yeast strains. The growth of the wild-type (full-length $B R F 1$ ) yeast strain and viable $B R F 1$ deletion yeast strains are shown at $18^{\circ} \mathrm{C}, 30^{\circ} \mathrm{C}$, and $37^{\circ} \mathrm{C} .(B)$ A summary of the viability and thermal sensitivity of the BRFl strains is shown with an accompanying graphic depiction of each deletion. The regions of similarity to TFIIB are indicated $(\boldsymbol{\square})$.
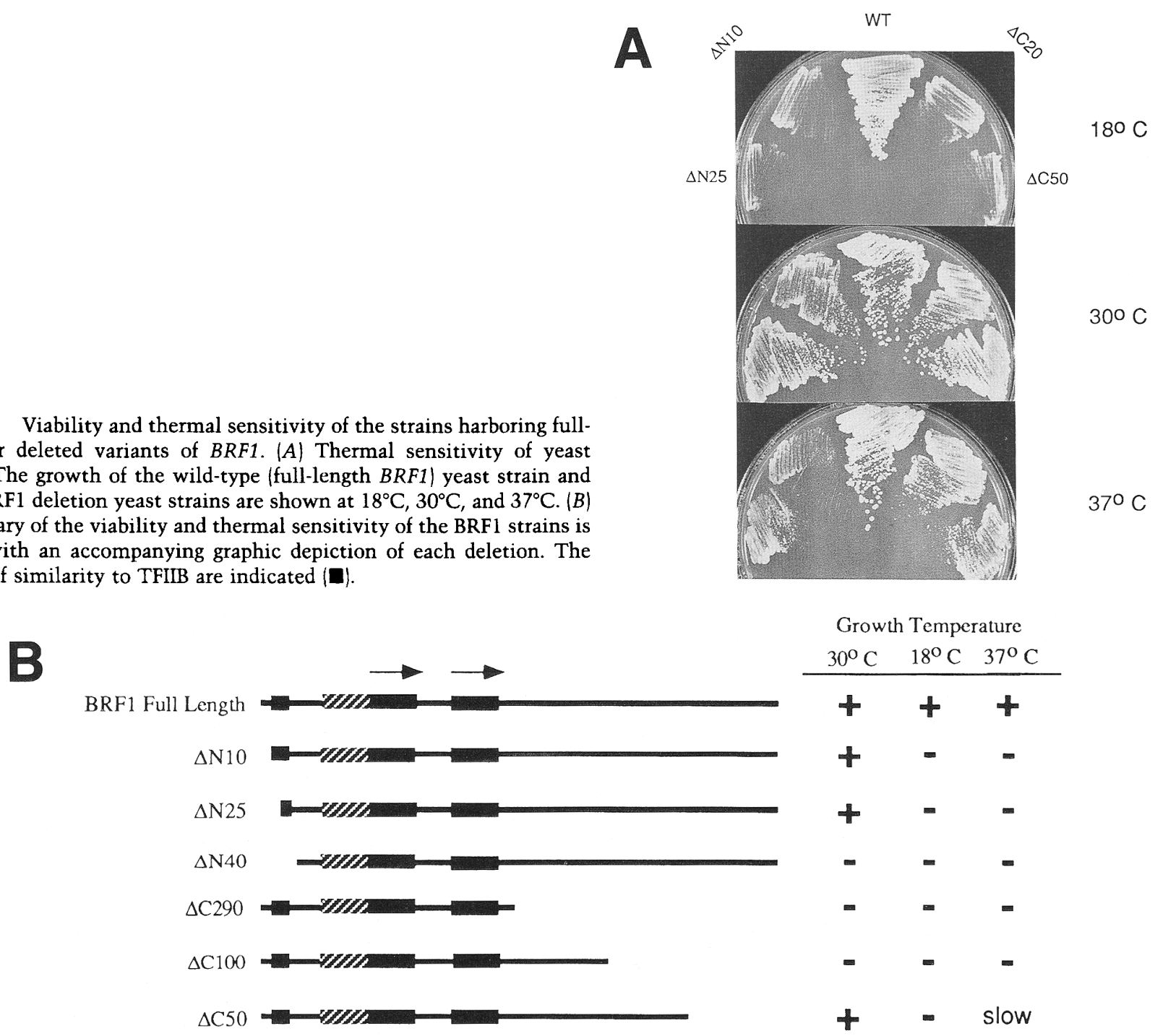

poorly, if at all, at either $18^{\circ} \mathrm{C}$ or $37^{\circ} \mathrm{C}$ compared with wild-type strains. Even at room temperature $\left(22^{\circ} \mathrm{C}\right)$, the BRF1 deletion strains grew poorly (data not shown).

\section{BRF1 is involved in RNA polymerase III transcription}

High-copy BRF1 suppresses the TBP P65S mutation, which is deficient in both Pol II and Pol III transcription in vitro. This suggests that BRFl is involved in RNA Pol II and/or RNA Pol III transcription. We used the conditional alleles of $B R F 1$ described above and the strategy of Schultz et al. (1992) to determine whether BRF1 affected Pol I, Pol II, or Pol III transcription. Nuclear and wholecell extracts were made from strains grown at $30^{\circ} \mathrm{C}$ containing either the wild-type or conditional alleles of $B R F 1$. We then tested in vitro whether Pol I, Pol II, or Pol III transcription was deficient in these extracts.

Pol III transcription was very sensitive to the absence of BRF1. Transcription from either the 5S rRNA gene
(Fig. 6A, top) or the SUP4 tRNA gene (Fig. 6A, bottom) was diminished three- to fourfold in the mutant BRF1 strains at the nonpermissive temperature of $22^{\circ} \mathrm{C}$ (Fig. $6 \mathrm{~A}$, cf. lane 1 with lanes 3,5 , or 7 , both top and bottom). Recombinant BRF1 was expressed and purified from Escherichia coli (Materials and methods). Recombinant BRF1 added to the wild-type extract stimulated transcription three- to fourfold from both the 5S and tRNA promoters showing that it is normally limiting for transcription in vitro (Fig. 6A, lane 2). The addition of BRF1 to the mutant extracts restored transcription to wildtype levels at both promoters. Similar results were seen when transcription was assayed at $37^{\circ} \mathrm{C}$. When assayed at $18^{\circ} \mathrm{C}$, little transcription was observed in the absence of added BRF1 from even the wild-type extract (data not shown). However, the addition of BRF1 at $18^{\circ} \mathrm{C}$ stimulated transcription from both mutant and wild-type extracts to levels equivalent to those shown in Figure 6A. These results indicate that BRF1 is involved in transcrip- 


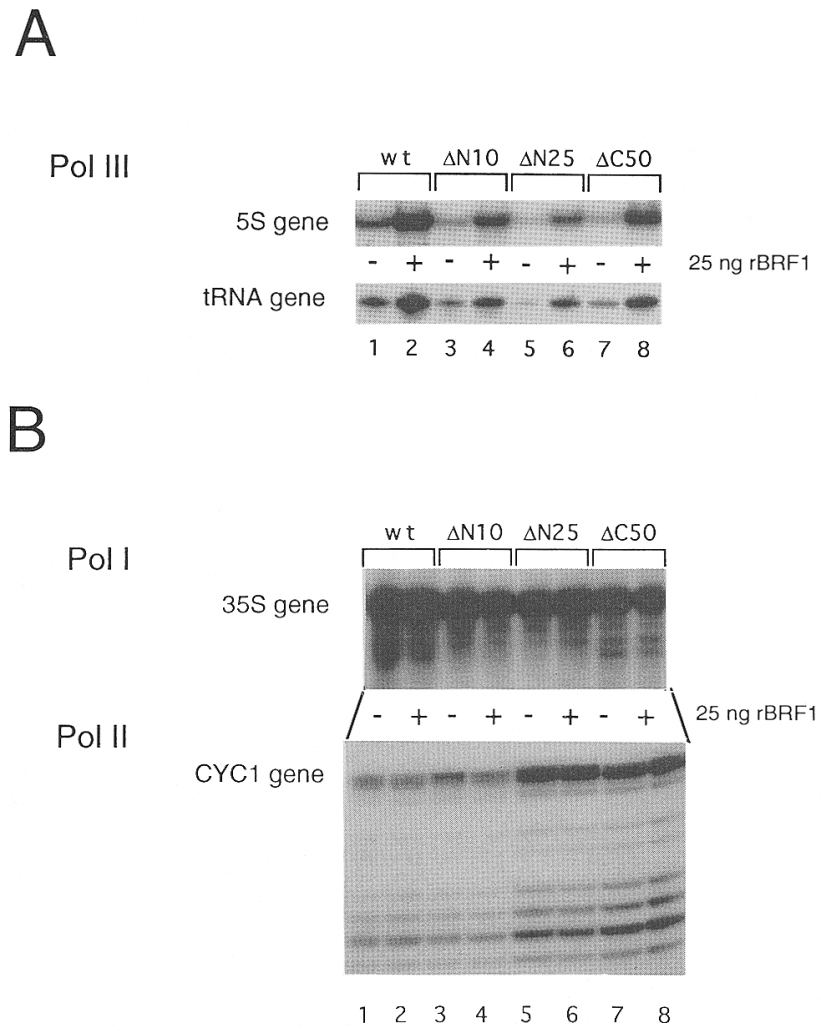

Figure 6. BRF1 is a Pol III-specific transcription factor. $(A)$ In vitro transcription by Pol III was assayed from the 5S rRNA gene (top) and SUP4 tRNA ${ }^{\text {tyr }}$ gene (bottom), using whole-cell extracts made from the indicated strains. Twenty-five nanograms of recombinant BRF1 (rBRF1) was added to each of the evennumbered lanes; no BRF1 was added to the odd-numbered lanes. (B) Pol I and Pol II transcription. The lane designations and the addition of recombinant BRFl in $B$ are as indicated in $A$. (Top) In vitro transcription from the $35 \mathrm{~S}$ rRNA gene, using whole-cell extracts made from the indicated strains. (Bottom) In vitro transcription was assayed from the CYC1 promoter using yeast nuclear extracts made from the indicated strains.

tion from the $5 \mathrm{~S}$ and tRNA genes and suggests that BRF1 is a Pol III general initiation factor.

In contrast to the results for Pol III transcription, Pol I transcription from the $35 \mathrm{~S}$ rRNA promoter was unaffected by mutations in BRF1. Mutant or wild-type extracts gave comparable levels of $\mathrm{Pol} \mathrm{I}$ initiation at $22^{\circ} \mathrm{C}$ (Fig. 6B top, cf. lane 1 with lanes 3,5 , and 7). Furthermore, the addition of recombinant $B R F 1$ protein did not stimulate Pol I transcription (Fig. 6B top, even-numbered lanes). Essentially the same results were observed when transcription was performed at $18^{\circ} \mathrm{C}$ and $37^{\circ} \mathrm{C}$ /data not shown).

In similar assays, transcription at $22^{\circ} \mathrm{C}$ by Pol II was examined at a promoter containing the yeast CYC1 TATA and initiation region with transcription driven by the GAL4-VP16 activator. As was observed with Pol I, Pol II transcription was unaffected by mutation in BRF1 (Fig. 6B bottom, cf. lane 1 with lanes 3,5 , or 7). Furthermore, the addition of recombinant BRF1 did not stimu- late Pol II transcription (Fig. 6B bottom, even-numbered lanes). Assays at $18^{\circ} \mathrm{C}$ gave similar results. At $37^{\circ} \mathrm{C}$ transcription by Pol II did not occur in vitro from either wild-type or mutant extracts (data not shown). The above results for Pol II are suprising given that BRF1 suppresses a TBP mutation defective in Pol II transcription. To confirm that BRF1 had no effect on Pol II transcription, we measured Pol II basal transcription as well as transcription from a promoter containing the $D E D 1$ upstream activating sequence (UAS), which responds to a weaker transcriptional activator than GAL4-VP16. Basal transcription from the CYC1 promoter lacking any transcriptional activator-binding sites failed to reveal any BRF1 effect on basal transcription. The DED1 UASdriven promoter also showed no BRF1 dependence (data not shown). Thus, under our assay conditions, BRF1 does not seem to be involved in Pol II transcription.

\section{BRF1 is a component of TFIIIB}

All of the factors required for RNAP III transcription are contained in TFIIIA, TFIIIB, and TFIIIC (for reference, see introductory section). Because TFIIIA is a single, wellcharacterized polypeptide, BRF1 could be a component of TFIIIB and/or TFIIIC. To test these possibilities, antibodies to BRF1 were generated in rabbits, affinity purified on a BRF1 protein column, and used to probe Western blots containing TFIIIB and TFIIIC /highly purified TFIIIB and TFIIIC were the generous gift of George Kassavetis). A single cross-reacting polypeptide of $\sim 70 \mathrm{kD}$ was detected in the TFIIIB fraction using Western analysis (Fig. 7A, lane 3). This cross-reacting band migrates to the same position as full-length recombinant BRF1 (Fig. 7A, lane 1) and the major immunoreactive band in a yeast nuclear extract (Fig. 7A, lane 2). Protein-DNA cross-linking has demonstrated that the $\mathrm{B}^{\prime}$ component of TFIIIB contains a $70-\mathrm{kD}$ polypeptide factor that is crosslinked to the SUP4 tRNA gene (Kassavetis et al. 1991). No BRF1 cross-reacting polypeptides were detected in TFIIIC (Fig. 7A, lane 4).

If TFIIIB contains active BRF1, then it should complement the BRF1-deficient extracts for Pol III transcription. As expected, the addition of TFIIIB to the wild-type and $\Delta N 25$ yeast extract stimulates transcription $\sim 7-$ to 10-fold (Fig. 7B; cf. lanes 1 and 4 with lanes 2 and 6), whereas the addition of TFIIIC does not enhance transcription of the mutant or wild-type extracts (Fig. $7 \mathrm{~B}$; lanes 3,7$)$. These data indicate that BRF1 is a component of TFIIIB.

\section{Discussion}

Suppression of a conditional TBP allele led to the isolation of a TFIIB-related factor, BRF1, involved in RNA Pol III transcription. Suppression of the temperature-sensitive defect by overproduction of BRF1 could occur either by stabilizing the structure or active conformation of TBP or by forcing the interaction between BRFl and TBP, which may have been weakened by the temperature-sensitive mutation. When BRF1 is in high copy 


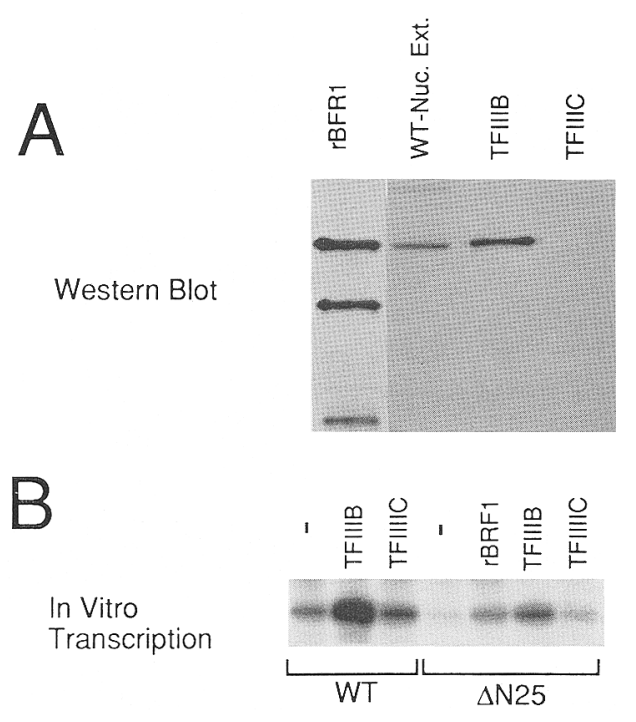

Figure 7. BRFl is a component of TFIIIB. (A) Western analysis of protein fractions using an affinity-purified anti-BRFl antibody. (Lane 1) Purified recombinant BRF1; (lane 2) $20 \mu \mathrm{g}$ of nuclear extract protein from wild-type yeast cells; (lane 3) 45 fmoles of TFIIIB; (lane 4) 45 fmoles of TFIIIC. Recombinant BRF1 was purified as described in Materials and methods. The three polypeptides seen in the recombinant BRF1 lane correspond to full-length BRF1 and two amino-terminal deletions of BRF1, which are also seen on a silver-stained gel. $(B)$ In vitro transcription from the $5 \mathrm{~S}$ rRNA promoter at $22^{\circ} \mathrm{C}$. Extracts were made from either wild-type or $\triangle \mathrm{N} 25 \mathrm{BRF} 1$ strains, and reactions contained either 10 fmoles of TFIIIB or TFIIIC, $25 \mathrm{ng}$ of recombinant BRF1, or no addition $(-)$.

number, it suppresses the temperature-sensitive phenotype of the TBP P65S mutation almost completely. This suppression is allele specific for TBP. A separate temperature-sensitive TBP allele, I143N, is not suppressed by BRF1 overproduction. This allele-specific suppression provides strong genetic evidence for the interaction of BRF1 and TBP. Furthermore, in agreement with the conclusions of Schultz et al. (1992), this suppression suggests that TBP has discrete regions that interact with polymerase-specific factors.

Although involved in Pol III transcription, we were not able to demonstrate that BRF1 was involved in Pol II transcription. Deletions of BRF1 from either the amino or carboxyl terminus decreased Pol III transcription specifically. Furthermore, BRF1 could not substitute for yeast SUA7 protein in a fractionated Pol II system (J. Ranish, unpubl.). These results seem surprising because BRF1 suppresses the TBP mutation shown previously to be defective in both Pol II and Pol III transcription in vitro. One possibility is that BRF1 is involved in Pol II transcription, but the deletions tested did not abolish its Pol II function. This possibility seems unlikely because deletions from either end of the protein affect only Pol III transcription. A second possibility is that BRF1 may associate with TBP in solution and stabilize the temperature-sensitive protein in an active state allowing it to promote both Pol II and Pol III transcription. We believe that second possibility is unlikely because recombinant BRFl cannot restore wild-type levels of Pol II transcription in P65S mutant extracts. In contrast, recombinant BRF1 will restore Pol III transcription when added to the mutant P65S extract (data not shown). A third possibility that we favor is that the P65S mutation is defective for only Pol III transcription in vivo. Consistent with this hypothesis, the $\mathrm{P} 65 \mathrm{~S}$ mutation shows some residual Pol II transcription in vitro compared with the I143N TBP mutation defective in all three nuclear polymerase activities (Schultz et al. 1992). Compared with wild-type extracts, the P65S mutant extracts are defective in Pol II transcription at low concentrations of nuclear extract. However, at high levels of nuclear extract in vitro, levels of Pol II transcription approach those seen in wild-type extracts (data not shown).

Extracts deficient in BRF1 are defective for Pol III transcription, and this defect is rescued by the addition of recombinant BRF1. Western analysis indicates that BRF1 is present in TFIIIB and not TFIIIC. This strongly suggests that BRF1 is likely to be an essential component of the previously defined TFIIIB fraction. Circumstantial evidence suggests that $\mathrm{BRF} 1$ is the $70-\mathrm{kD}$ polypeptide contained in the $\mathrm{B}^{\prime}$ fraction that was shown to cross-link to the SUP4 tRNA promoter. First, BRF1 chromatographs similarly to $\mathrm{B}^{\prime}$ when applied to DEAE, Mono-S, and Cibacron blue (T. Colbert, unpubl.). Second, $B^{\prime}$ footprints and cross-links in the vicinity of the transcription start site (Kassavetis et al. 1989; Bartholomew et al. 1990; Kassavetis et al. 1991), in an analogous position to TFIIB in the Pol II system (Buratowski et al. 1989; Maldonado et al. 1990). Finally, our purified antibodies to BRF1 supershift a TFIIIB-promoter complex /G. Kassavetis, pers. comm.l, indicating that BRF1 is part of the TFIIIB stable complex at a Pol III promoter.

Western analysis of fractionated nuclear extracts $/ T$. Colbert, unpubl.) showed that BRF1 chromatographs heterogeneously, with the bulk of BRF1 appearing in fractions I and II of the fractionation scheme described by Ranish et al. (1992). Because fraction II also contains the bulk of TBP (Ranish et al. 1992), it seems that TBP is likely to be a component of the fraction defined as TFIIIB. On the basis of the genetic evidence for the interaction between BRF1 and TBP, we propose that the BRF1-TBP interaction stabilizes the interaction of TBP at Pol III promoters because TBP itself has weak affinity for DNA lacking a TATA element (Hahn et al. 1989; Wobbe and Struhl 1990). This arrangement of factors along the DNA might be analogous to the Pol II system where TBP almost always binds upstream of TFIIB. In Pol III promoters containing a TATA element, such as the vertebrate U6 gene, TBP bound to a TATA element could bring TFIIIB to the promoter to form a stable initiation complex through the interaction with a vertebrate BRFl-like factor. In this situation, tightly bound TBP would replace the need for TFIIIC, which normally recruits TFIIIB into the initiation complex /similar hypotheses are invoked by Dahlberg and Lund 1991; Lobo et al. 1991). Subtle differences in the TATA element are known to change the polymerase specifity between Pol II 
and Pol III (Mattaj et al. 1988; Lobo and Hernandez 1989). This may be the result of an alteration in TBP activity, which changes its specificity for interaction with TFIIB versus a BRF1-like factor.

The identification of BRF1 adds to the evidence that initiation by the three nuclear polymerases occurs by similar mechanisms. In the Pol II system, the binding of TFIIB to the initiation complex occurs just before the association of PolII-TFIIF (Flores et al. 1991; Zawel and Reinberg 1992). Because BRF1 is homologous with TFIIB, it is likely that BRF1 plays an analogous role and interacts with the Pol III enzyme leading to stable complex formation. Because both yeast Pol II and Pol III use TFIIB-related factors, it will be interesting to address whether a Pol I general factor is also related to TFIIB, and also whether other eukaryotes utilize multiple TFIIBrelated factors.

\section{Materials and methods}

\section{Isolation of BRF1}

To isolate high-copy suppressors of the temperature-sensitive TBP mutations, a high-copy $2 \mu$ yeast genomic library (Carlson and Botstein 1982) was transformed to the two temperaturesensitive strains and plated on minimal glucose plates at the permissive temperature of $25^{\circ} \mathrm{C}$. Transformants were scraped from these plates and replated on minimal glucose plates at both $25^{\circ} \mathrm{C}$ and $37^{\circ} \mathrm{C}$. Revertants that grew at $37^{\circ} \mathrm{C}$ were picked, and plasmid DNA was isolated by insertion into $E$. coli. This DNA was retransformed into yeast to confirm the temperaturesensitive suppression as well as checked by restriction digest to determine whether the suppressor contained the SPT15 gene.

For DNA sequencing, the ClaI fragment of BRF1 was cloned into a Bluescript plasmid (Stratagene), and directional deletions were constructed using exonuclease III (Promega, Erase-a-base kit). The DNA sequence was determined on both strands using dideoxy sequencing.

\section{Disruption of BRF1}

Disruption of $B R F 1$ was accomplished by replacing the sequences between BgIII and StuI with the yeast HIS4 gene. This disruption deletes amino acids $15-287$ in the BRF1 ORF. The resulting DNA fragment was used to transform a diploid strain to His ${ }^{+}$. BRF1 disruption was confirmed by Southern analysis. Diploids were sporulated and spores were tested for the His ${ }^{+}$ phenotype. To construct a haploid strain containing $B R F 1$ on a plasmid with the chromosomal copy disrupted, the diploid with one chromosome disrupted was transformed with a URA3marked plasmid containing $B R F 1$. This strain was sporulated as described above to generate the desired strain. Using this strain, the BRF1 deletions generated by polymerase chain reaction (PCR) and carried on an Ars-Cen LEU2 plasmid were substituted for wild-type BRF1 by plasmid shuffle on FOA plates.

\section{Similarity searches}

The regions of similarity between TFIIB-related proteins were found using the MACAW program created by the National Center for Biotechnology Information (Schuler et al. 1991).

The RSS program of the FASTA group of programs (Pearson and Lipman 1988) compares a group of shuffled alignments with that of the Lipman and Pearson alignment algorithm (Lipman and Pearson 1985). Comparison with shuffled sequences allows one to "subtract" any similarity that is the result of common structural motifs and/or repetitive sequences, for example, a high content of any particular amino acid. The proteins were shuffled in 10-amino-acid blocks to reflect these structural conservations and repetitive sequences. The relatedness of two sequences is expressed as the number of standard deviations by which the Lipman and Pearson alignment score differs from the mean alignment score of 100 alignments with a randomly shuffled sequence. Scores greater than 6 S.D.s above the mean suggest that the sequences (Pearson 1990) are related.

\section{BRF1 deletion mutations}

A plasmid carrying the genomic clone of BRF1 on a ClaI fragment was inserted into pBlueScript II SK + and mutagenized by site-directed mutagenesis. An oligonucleotide (WT 5') was used to mutagenize the initial methionine codon to methionine-alanine and also inserted a $\mathrm{NCOI}$ site at the $5^{\prime}$ end of the BRF1coding region. Mutagenesis was confirmed by DNA sequencing. Oligonucleotides corresponding to the various deletions were synthesized and purified on polyacrylamide-urea gels. The $5^{\prime}$ oligonucleotides insert the same $\mathrm{NcoI}$ site and alanine as the site-directed mutagenesis. The 3' oligonucleotides contained a translation stop codon (UAA) and a SacI site. Deletion oligonucleotides are given below. Restriction sites, alanine insertion, and stop codons are underlined. WT 5', 5'-AGTCATATACCATGGCTCCAGTGTGTAAG- ${ }^{\prime}$; N10, 5'-TCAGCGCCATGGCTGAGAGAGATCTTTCCAATGC-3'; N25, 5'-TCAGCGCCATGGCTTGTGGTGTTGTCTCAGAAG-3'; N40, 5' -TCAGCGCCATGGCTTTCGGTGAAACAAGTGCAGG-3'; C290, 5' - TCAGCGGAGCTCTTATTCTTTGTCTAAAGAGTCC-3'; C100, 5'-TCAGCGGAGCTCTTAATTCAAGTAAGAAATCTGCG-3'; C50, 5'-TCAGCGGAGCTCTTAGTCTGACATCAATCCGATAGC-3'; C20, 5'-TCAGCGGAGCTCTTACAACATGTTCTTGACACTATC-3'; WT 3', 5'-TCAGCGGAGCTCT. TACCTAAACAAACCGTC-3'.

The above oligonucleotides were used to amplify (by PCR) the BRF1 fragments with Vent polymerase (New England Biolabs) using the manufacturer's buffer and recommendations. The fragments were cloned into the vectors pRS315(Cen-Ars, Leu2) and YEP351 $(2 \mu$, Leu2 $)$, and the junctions were confirmed by sequencing. These constructs were shuffled into yeast by the method mentioned above.

\section{Production of recombinant BRF1}

The coding region of the $B R F 1$ gene was subcloned into the plasmid pet-11d (Novagen) by PCR amplification (as above) using the WT-5' oligonucleotide and the SmaI-containing oligonucleotide which also added 6 histidines to the carboxyl terminus of BRF1.

Expression of the BRF1- 6 His protein was driven by the T7 promoter in $E$. coli harboring the T7 polymerase under Lac control. For details of induction, see Ranish et al. (1992). The BRF1 protein was purified from 70 grams of cells under denaturing conditions on $\mathrm{a} \mathrm{Ni}^{+}$column as described by the column's manufacturer (Qiagen Corp.). BRFl was renatured by $1: 1$ dilution from the column elution buffer into $50 \mathrm{mM}$ Tris-acetate, 150 $\mathrm{mM} \mathrm{K}$ acetate, $5 \mathrm{mM} \mathrm{DTT}$, and $5 \mathrm{~mm} \mathrm{Mg}$-acetate plus protease inhibitors, and dialysis against the same buffer. The concentrations of the protease inhibitors were as described by Ranish et al. (1992). BRF1 was further purified by application to a Mono-S column in the renaturation buffer described above and developed with a $150 \mathrm{mM}$ to $1 \mathrm{M} \mathrm{K}$ acetate gradient. BRF1 eluted at $\sim 500 \mathrm{mM} \mathrm{K}$ acetate. The total yield of BRFl was estimated to be 
$15 \mathrm{mg}$. Following SDS-PAGE, $\sim 60 \%$ of the BRF1 was full-length and the other $40 \%$ of the BRF1 protein was contained in two proteolytic fragments of 60 and $30 \mathrm{kD}$, respectively. No stainable contaminants were detected.

\section{Antibody production and Western blot analysis}

Three New Zealand White, female rabbits were immunized with $50 \mu \mathrm{g}$ of 6 His-BRF1 protein and boosted twice before the first bleed. Antiserum was affinity purified on an Affi-gel 10 BRF1 column. Briefly, $5 \mathrm{mg}$ of 6 His-BRF1 was coupled to an Affi-gel 10 (Bio-Rad) column in $50 \mathrm{~mm}$ MOPS (pH 7.0), $100 \mathrm{~mm}$ $\mathrm{NaCl}$ for $1 \mathrm{hr}$ at $4^{\circ} \mathrm{C}$, reduced with $1 \mathrm{M}$ triethanolamine, and washed with MOPS buffer plus $1 \mathrm{mg} / \mathrm{ml}$ of BSA. The column was equilibrated in TBS [50 $\mathrm{mM}$ Tris acetate, $150 \mathrm{mM}\left(\mathrm{NH}_{4}\right)_{2} \mathrm{SO}_{4}$ [pH 7.9) $]$ and loaded with a $1: 1$ dilution of serum and TBS. The column was washed with TBS, and the antibody was eluted with $0.1 \mathrm{M}$ acetic acetate $(\mathrm{pH} 2.5)$. The eluted antibody was adjusted immediately to $\mathrm{pH} 7.5$ with $2 \mathrm{M}$ Tris following elution. The protein concentration of the antibody solution was determined by the the method of Bradford (Bio-Rad).

Western blots were performed by transferring SDS-PAGE-separated proteins to Immobilon membranes (Millipore), essentially as in Harlow and Lane (1988). The amount of recombinant BRFl loaded onto the gel was $250 \mathrm{ng}, \sim 10$ times the amount of $B R F 1$ as in the yeast extract; thus, the recombinant BRF1 lane (Fig. 7) was exposed to film for less time than the others. The concentrations of the other protein samples are indicated in Figure 7. Nonspecific interaction of the antibody was prevented by blocking for $2 \mathrm{hr}$ in $50 \mathrm{~mm}$ Tris- $\mathrm{HCl}, 150 \mathrm{~mm} \mathrm{NaCl}, 0.05 \%$ Tween-20 (pH 8.0) (TBST), plus 7.5\% nonfat dry milk. The affinity-purified anti-BRFl antibody was diluted into the blocking solution to a final concentration of $200 \mathrm{pg} / \mathrm{ml}$ and incubated for $1 \mathrm{hr}$ with the membrane. The membrane was washed in TBST, incubated in a $1: 5000$ dilution of horseradish peroxidase-conjugated anti-rabbit (Fc) IgG antibody (Promega) for $1 \mathrm{hr}$, and washed again in TBST. Protein bands recognized by the antiBRF1 antisera were developed by Enzyme-linked Chemiluminescence (ECL, Amersham).

\section{Transcription assays}

Pol I transcription was performed using whole-cell extracts (Schultz et al. 1991) under the conditions of Schultz et al. (1992). Assays were also done with yeast nuclear extracts as the source of protein. Yeast nuclear extracts were prepared as in Ranish et al. (1992). The amount of template was $20 \mu \mathrm{g} / \mathrm{ml}$, the protein content (either whole-cell extracts or nuclear extracts) was 45 $\mu \mathrm{g}$ per reaction, the reaction volume was $20 \mu \mathrm{l}$, and the incubation time was $30 \mathrm{~min}$ at room temperature. Transcription was done in the presence of $10 \mu \mathrm{g} / \mathrm{ml}$ of $\alpha$-amanatin. Pol II transcription was performed using yeast nuclear extracts as the source of protein, and all assay conditions were as in Ranish et al. (1992). Pol III transcription was performed as in Schultz et al. (1992) with both whole-cell and nuclear extracts as the source of protein, with the exception that tRNA transcription was from the SUP4 gene on pTZ1 (Kassavetis et al. 1989). All other reaction conditions were identical to Pol I transcription.

\section{Acknowledgments}

We thank David Auble, Jeff Ranish, and George Kassavetis for critical comment, encouragement, and permission to cite unpublished observations. We also thank Peter Geiduschek, Sally Lee, Daniel Miller, Phillipe Szankasi, Ron Reeder, and Mark
Groudine for their comments on the manuscript and their general encouragement. We thank Kurt Runge for the yeast library and for helpful advice, and Jim Kadonaga for sharing the Drosophila TFIIB sequence prior to publication. Steve Henikoff gave us valuable advice on the usefulness and pitfalls of sequence comparisons. T.C. thanks the faculty at Grinnell College for their enthusiasm and support throughout his undergraduate years. The research in this paper was supported by grants to S.H. from the National Institutes of Health and and an American Cancer Society Junior Faculty Award and by a U.S. Public Health Service training grant awarded to T.C.

The publication costs of this article were defrayed in part by payment of page charges. This article must therefore be hereby marked "advertisement" in accordance with 18 USC section 1734 solely to indicate this fact.

\section{References}

Bartholomew, B., G.A. Kassavetis, B.R. Braun, and E.P. Geiduschek. 1990. The subunit structure of Saccharomyces cerevisiae transcription factor IIIC probed with a novel photocross-linking agent. EMBO J. 9: 2197--2205.

Bartholomew, B., G.A. Kassavetis, and E.P. Geiduschek. 1991. Two components of Saccharomyces cerevisiae transciption factor IIIB (TFIIIB) are stereospecifically located upstream of a tRNA gene and interact with the second-largest subunit of TFIIIC. Mol. Cell. Biol. 11: 5181-5189.

Bell, S.P., R.M. Learned, H.M. Jantzen, and R. Tjian. 1988. Functional cooperativity between transcription factors UBF and SLl mediates human ribosomal RNA synthesis. Science 241: 1192-1197.

Braun, B.R., D.L. Riggs, G.A. Kassavetis, and E.P. Geiduschek. 1989. Multiple state of protein-DNA interaction in the assembly of transcription complexes on Saccharomyces cerevisiae 5S ribosomal RNA genes. Proc. Natl. Acad. Sci. 86: $2530-2534$.

Buratowski, S., S. Hahn, L. Guarente, and P.A. Sharp. 1989. Five intermediate complexes in transcription initiation by RNA polymerase II. Cell 56: 549-561.

Carles, C., I. Treich, F. Bouet, M. Riva, and A. Sentenac. 1991. Two additional common subunits, $\mathrm{ABC} 10 \alpha$ and $\mathrm{ABC10} \beta$, are shared by yeast RNA polymerases. I. Biol. Chem. 266: 24092-24096.

Carlson, M. and D. Botstein. 1982. Two differentially regulated mRNAs with different $5^{\prime}$ ends encode secreted and intracellular forms of yeast invertase. Cell 28: 145-154.

Comai, L., N. Tanese, and R. Tjian. 1992. The TATA-binding protein and associated factors are integral components of the RNA polymerase I transcription factor, SL1. Cell 68: 965976.

Cormack, B.P. and K. Struhl. 1992. The TATA-binding protein is required for transcription by all three nuclear RNA polymerases in yeast cells. Cell 69: 685-696.

Dahlberg, J.E. and E. Lund. 1991. How does III $\times$ II make U6? Science 254: 1462-1463.

Flores, O., H. Lu, M. Killeen, J. Greenblatt, Z.F. Burton, and D. Reinberg. 1991. The small subunit of transcription factor IIF recruits RNA polymerase II into the preinitiation complex. Proc. Natl. Acad. Sci. 88: 9999-10003.

Gabrielson, O.S. and A. Sentenac. 1991. RNA polymerase III(C) and its transcription factors. Trends Biochem. Sci. 16: 412416.

Geiduschek, E.P. and G.A. Kassavetis. 1992. RNA polymerase III transcription complexes. In Transcriptional regulation. Cold Spring Harbor Laboratory Press, Cold Spring Harbor, 
New York (In press.)

Geiduschek, E.P. and G.P. Tocchini-Valentini. 1988. Transcription by RNA polymerase III. Annu. Rev. Biochem. 57: 873914.

Ginsberg, A.M., B.O. King, and R.G. Roeder. 1984. Xenopus 5S gene transcription factor, TFIIIA: Characterization of a cDNA clone and measurement of RNA levels throughout development. Cell 39: 479-489.

Ha, I., W.S. Lane, and D. Reinberg. 1991. Cloning of a human gene encoding the general transcription initiation factor IIB. Nature 352: 689-695.

Hahn, S., S. Buratowski, P.A. Sharp, and L. Guarente. 1989. Yeast TATA-binding protein TFIID binds to TATA elements with both consensus and non-consensus DNA sequences. Proc. Natl. Acad. Sci, 86: 5718-5722.

Harlow, E. and D. Lane. 1988. Antibodies: A laboratory manual. Cold Spring Harbor Laboratory, Cold Spring Harbor, New York.

Hayles, J., D. Beach, B. Durkacz and P. Nurse. 1986. The fission yeast cell cycle control gene $c d c 2$ : Isolation of a sequence suc1 that suppresses cdc2 mutant function. Mol. Gen. Genet. 202: 291-293.

Hisatake, K., S. Malik, R.G. Roeder, and M. Horikoshi. 1991. Conserved structural motifs between Xenopus and human TFIIB. Nucleic Acids Res. 19: 6639.

Kassavetis, G.A., D.L. Riggs, R. Negri, L.H. Nguyen, and E. Geiduschek. 1989. Transcription factor IIIB generates extended DNA interactions in RNA polymerase III transcription complexes on tRNA genes. Mol. Cell. Biol. 9: 2551-2566.

Kassavetis, G.A., B. Bartholomew, J.A. Blanco, T.E. Johnson, and E.P. Geiduschek. 1991. Two essential components of the Saccharomyces cerevisiae transcription factor TFIIIB: Transcription and DNA-binding properties. Proc. Natl. Acad. Sci. 88: $7308-7312$.

Learned, R.M., S. Cordes, and R. Tjian. 1985. Purification and characterization of a transcription factor that confers promoter specificity to human RNA polymerase I. Mol. Cell. Biol. 5: 1358-1369.

Lipman, D.J. and W.R. Pearson. 1985. Rapid and sensitive protein similarity searches. Science 227: 1435-1441.

Lobo, S.M. and N. Hernandez. 1989. A 7bp mutation converts a human RNA polymerase II snRNA promoter into an RNA polymerase III promoter. Cell 58: $55-67$.

Lobo, S.M., J. Lister, M.L. Sullivan, and N. Hernandez. 1991. The cloned RNA polymerase II transcription factor IID selects RNA polymerase III to transcribe the human U6 gene in vitro. Genes \& Dev. 5: 1477-1489.

Maldonado, E., I. Ha, P. Cortes, L. Weis, and D. Reinberg. 1990. Factors involved in specific transcription by mammalian RNA polymerase II: Role of transcription factors IIA, IID, and IIB during formation of a transcription-competent complex. Mol. Cell. Biol. 10: 6335-6347.

Malik, S., K. Hisatake, H. Sumimoto, M. Horikoshi, and R.G. Roeder. 1991. Sequence of general transcription factor TFIIB and relationships to other initiation factors. Proc. Natl. Acad. Sci. 88: 9553-9557.

Margottin, F., G. Dujardin, M. Gerard, J.-M. Egly, J. Huet, and A. Sentenac. 1991. Participation of the TATA factor in transcription of the yeast U6 gene by RNA polymerase C. Science 251: 424-426.

Mattaj, I.W., N.A. Dathan, H.D. Parry, P. Carbon, and A. Krol. 1988. Changing the RNA polymerase-specificity of U snRNA gene promoters. Cell 55: 435-442.

Parson, M.C. and P.A. Weil. 1992. Cloning of TFCl, the Saccharomyces cerevisiae gene encoding the $95 \mathrm{kDa}$ subunit of TFIIIC. I. Biol. Chem. 267: 2894-2901.
Pearson, W.R. and D.J. Lipman. 1988. Improved tools for biological sequence comparison. Proc. Natl. Acad. Sci. 85: 2444-2448.

Pearson, W.R. 1990. Rapid and sensitive sequence comparison with FASTP and FASTA. Methods Enzymol. 183: 63-98.

Pinto, I., D.E. Ware, and M. Hampsey. 1992. The yeast SUA7 gene encodes a homolog of human transcription factor TFIIB and is required for normal start site selection in vivo. Cell 68: $977-988$

Ranish, J.A., W.S. Lane, and S. Hahn. 1992. Isolation of two genes that encode subunits of the yeast transcription factor IIA. Science 255: 1127-1129.

Reeder, R.H. 1992. The regulation by RNA polymerase I initiation. In Transcriptional regulation. Cold Spring Harbor Laboratory Press, Cold Spring Harbor, New York (In press.)

Schuler, G.D., S.F. Altschul, and D.J. Lipman. 1991. A workbench for multiple alignment construction and analysis. Proteins Struct. Func. Genet. 9: 180-190.

Schultz, M.C., S.Y. Choe, and R.H. Reeder. 1991. Specific initiation by RNA polymerase $I$ in a whole-cell extract from yeast. Proc. Natl. Acad. Sci. 88: 1004-1008.

Schultz, M.C., R.H. Reeder, and S. Hahn. 1992. Variants of the TATA-binding protein can distinguish subsets of RNA polymerase I, II, and III. Cell 69: 697-702.

Sentenac, A. 1985. Eukaryotic RNA polymerases. CRC Crit. Rev. Biochem. 18: 31-90.

Simmen, K.A., J. Bernues, H.D. Parry, H.G. Stunnenberg, A. Berkenstam, B. Cavallini, J.-M. Egly, and I.W. Mattaj. 1991. TFIID is required for in vitro transcription of the human U6 gene by RNA polymerase III. EMBO J. 10: 1853-1862.

Sollner-Webb, B. and E.B. Mougley. 1991. News from the nucleolus: rRNA gene expression. Trends. Biochem. Sci. 16: $58-62$.

Swanson, R.W., C. Conesa, O. Lefebvre, C. Carles, A. Roiet, E. Quemeneur, J. Gagon, and A. Sentenac. 1991. Isolation of TFCl a gene encoding one of the two DNA binding subunits of yeast transcription factor tau (IIIC). Proc. Natl. Acad. Sci. 88: $4887-4891$.

White, R.J., S.P. Jackson, and P.W.J. Rigby. 1992. A role for the TATA-box-binding protein component of the transcription factor IID complex as a general RNA polymerase III transcription factor. Proc. Natl. Acad. Sci. 89: 1949-1953.

Wobbe, C.R. and K. Struhl. 1990. Yeast and human TATA-binding proteins have nearly identical DNA sequence requirements for transcription in vitro. Mol. Cell. Biol. 10: 38593867.

Woychik, N.A. and R.A. Young. 1992. Genes encoding trancription factor IIIA and the RNA polymerase common subunit RBP6 are divergently transcribed in Saccharomyces cerevisiae. Proc. Natl. Acad. Sci. 89: 3999-4003.

Woychik, N.A., S.-M. Liao, P.A. Kolodziej, and R.A. Young 1990. Subunits shared by eukaryotic nuclear RNA polymerases. Genes \& Dev. 4: 313-323.

Yamashita, S., K. Wada, M. Horikoshi, D.-W. Gong, T. Kokubo, K. Hisatake, N. Yokotani, S. Malik, R.G. Roeder, and Y. Nakatani. 1992. Isolation and characterization of a cDNA encoding Drosophila transcription factor TFIIB. Proc. Natl. Acad. Sci. 89: 2839-2843.

Young, R.A. 1991. RNA polymerase II. Annu. Rev. Biochem. 60: 689-715.

Zawel, L. and D. Reinberg. 1992. Initiation of transcription by RNA polymerase II: A multi-step process. Prog. Nucleic Acids Res. Mol. Biol. (in press). 


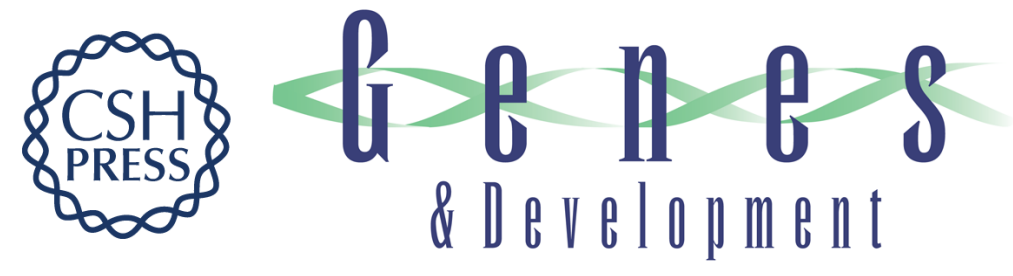

\section{A yeast TFIIB-related factor involved in RNA polymerase III transcription.}

T Colbert and S Hahn

Genes Dev. 1992, 6:

Access the most recent version at doi:10.1101/gad.6.10.1940

References This article cites 46 articles, 25 of which can be accessed free at:

http://genesdev.cshlp.org/content/6/10/1940.full.html\#ref-list-1

License

Email Alerting

Service

Receive free email alerts when new articles cite this article - sign up in the box at the top right corner of the article or click here.

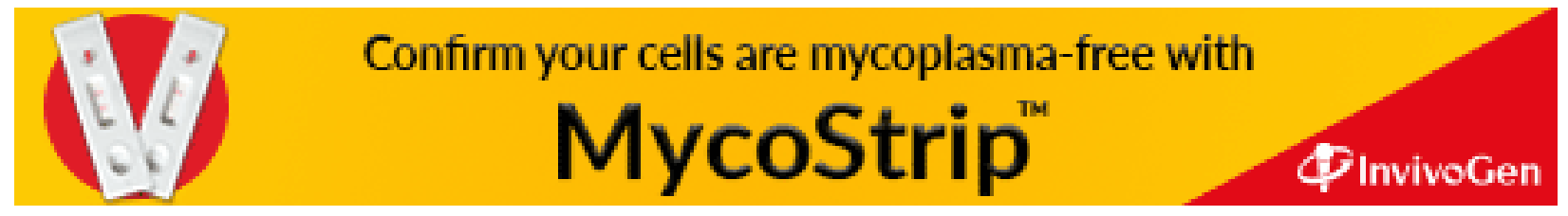

